\title{
Papel dos ácidos graxos ômega 3 na resolução dos processos inflamatórios
}

\section{Omega 3 fatty acid and the resolution of inflammatory processes}

\author{
Sandra M. Barbalho; Marcelo D. Bechara²; Karina R. Quesada; Ricardo A. Goulart ${ }^{4}$
}

\begin{abstract}
RESUMO
A inflamação é uma resposta de defesa do organismo ao trauma ou a infecções por microorganismos. $\mathrm{Na}$ fase de término da inflamação e retorno à homeostase ocorrem mecanismos de regulação chamados de resolução onde há alternância de prostaglandinas pró-inflamatórias e leucotrienos a mediadores pró-resolução, produzidos a partir de ácidos graxos poliinsaturados $\omega 3$ (ácido eicosapentanóico e ácido docosapentanóico). A dieta interfere na composição de ácidos graxos das membranas celulares que serão metabolizados em compostos ativos chamados de resolvinas, protectinas e maresinas. Como as doenças inflamatórias têm papel importante na saúde pública o estudo destas substâncias e o delineamento de seus efeitos podem trazer novos horizontes no tratamento das doenças inflamatórias, com redução de custos e minimização de efeitos colaterais.
\end{abstract}

Palavras-Chave: Inflamação. Ácidos Graxos Ômega-3. Resolvinas. Protectinas.

\section{Introdução}

O processo inflamatório é uma resposta de defesa ao trauma ou a infecções microbianas e é iniciada com a finalidade de extinguir o estímulo que a desencadeou ou para remover um dano tissular. Muitas doenças como mal de Alzheimer, Diabetes, doenças cardiovasculares, asma, artrite reumatóide, periodontite, câncer e outras são resultantes de processos inflamatórios inapropriados ou excessivos que as iniciam e as acompanham de forma crônica. ${ }^{1,2}$

Em condições favoráveis a resposta inflamatória aguda é adequada de tal forma que controla os eventos danosos ao tecido, por exemplo, para eliminar microorganismos invasores e para preparar o local para os processos de recuperação. Se os danos teciduais são de proporções tais que as células podem recuperar suas funções normais, dá-se o nome ao processo de resolução. No entanto, muitas vezes a lesão persiste e os mecanismos de cicatrização não se completam. A conseqüência é que são estimuladas as defesas do sistema imunológico com ativação de macrófagos, liberação de mediadores químicos, metabólitos reativos (radicais livres), moléculas de adesão, proteases e enzimas hidrolíticas, citocinas inflamatórias, como as interleucinas (IL) e fator de necrose tumoral $\alpha(\mathrm{TNF} \alpha)$ e fatores de crescimento. ${ }^{3-9}$

No caminho de término da inflamação em direção da homeostase, ou seja, no processo de resolução, ocorrem mecanismos de regulação ativa onde há
1. Professora de Bioquímica, Faculdade de Medicina de Marília UNIMAR- Marília/SP; Faculdade de Tecnologia de Alimentos de Marília/SP.

2.Professor de Imunologia e Biologia Molecular, Faculdade de Medicina de Marília - UNIMAR-Marília/SP.

3. Professora de Nutrição, Faculdade de Ciências da Saúde da UNIMAR Marília/SP e da UNIP-Assis/SP.

4. Médico, Faculdade de Medicina - FAMEMA - Marília/SP.
Correspondência: Sandra Maria Barbalho Av. Sampaio Vidal, 300 - apto. 24 - Bairro Barboza 17500021 - Marília - SP smbarbalho@terra.com.br Aprovado para publicação em 13/09/2011 
alternância de prostaglandinas pró-inflamatórias e leucotrienos (para a biossíntese de mediadores inflamatórios) a mediadores pró-resolução, produzidos em exsudatos a partir de ácidos graxos poliinsaturados (AGP) $\omega 3$ (ácido eicosapentanóico - EPA e ácido docosapentanóico - DHA). Os Neutrófilos, que inicialmente liberam mediadores inflamatórios, podem modificar o perfil dos produtos liberados passando a produzir mediadores lipídicos que funcionarão como agonistas na limitação do processo inflamatório., ${ }^{70-13}$

Estes ácidos graxos foram relacionados há muito tempo com a prevenção de doenças e o interesse nesta categoria de substâncias tem crescido enormemente nos últimos anos. Há vários estudos que indicam que a suplementação dietética com $\omega 3$ poliinsaturado pode ter impacto positivo na saúde de animais e seres humanos em diferentes doenças inflamatórias. ${ }^{7}, 13-16$ Entre os mecanismos que podem explicar os efeitos benéficos dos $\omega 3$ está a competição pelo substrato que previne a conversão de Ácido Araquidônico (AA) a eicosanóides pró-inflamatórios como as prostaglandinas (PTG), leucotrienos (LEU) e lipoxinas (LX) graças à cicloxigenase (COX) ou lipoxigenase (LOX) (Figura 1). Desta forma o $\omega 3$ funciona como um substrato que resulta em compostos menos potentes do que as PTG e LEU. Além disso, sabe-se que mediadores derivados destes $\omega 3$ estão envolvidos na produção de resolvinas e protectinas, importantes na resolução da inflamação. ${ }^{2,17}$

Os primeiros mediadores derivados do AA que foram descritos foram as LX, que exibem efeitos próinflamatórios e têm papel importante na resolução.
Estas e outras moléculas com efeitos similares foram designadas de resolvinas e protectinas. ${ }^{7,13,18,19}$

\section{Papel dos AGP $\omega 3$}

Os AGP $\omega 3$ podem ser encontrados em proporções substanciais em diferentes fontes alimentares, como é o caso da linhaça e peixes como o salmão. ${ }^{20}$

A relação destes ácidos graxos e os processos inflamatórios é que os eicosanóides são formados a partir de AG de 20 carbonos. Nas células com respostas inflamatórias há sempre altos níveis de AA (20:4 6) e baixos níveis de outros ácidos graxos. Sendo assim, o AA está sempre em maior disponibilidade como precursor para a biossíntese dos eicosanóides que estarão envolvidos na modulação da intensidade e duração das respostas inflamatórias. Os aspectos patofisiológicos de sua ação dependem do tecido alvo, da natureza do estímulo, da produção e quantidade de eicosanóides que são produzidos e da sensibilidade das células alvo à sua ação. ${ }^{1,21-24}$

Os AGP $\omega 3$ podem auxiliar na proteção de doença vascular aterosclerótica, artrite, pancreatite, alergias, pneumonia e outras doenças inflamatórias. Podem, por exemplo, modular o processo inflamatório dentro da parede do vaso sanguíneo alterando também a composição estrutural e celular da placa aterosclerótica em estado avançado de tal forma que pode reduzir a ruptura ou ulceração da placa, que é um processo que precede o infarto do miocárdio. ${ }^{7,12,15}$, $19,24,25,26$
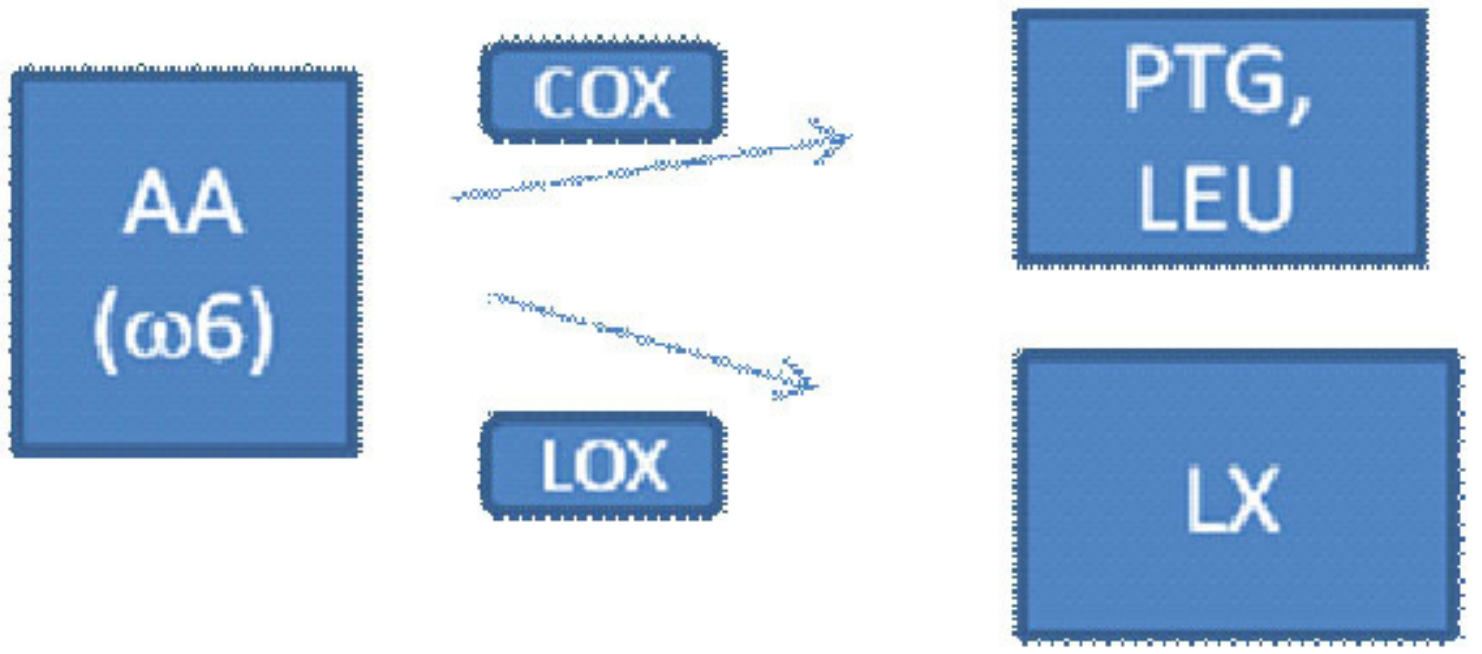

Figura 1. Formação de prostaglandinas (PTG) e leucotrienos (LEU) via ciclo-oxigenase (COX) e lipoxinas (LX) via lipoxigenase (LOX). Adaptado de SERHAN, CHIANG, 2008. 
Os AGP $\omega 3$ (EPA e DHA) têm efeitos similares aos das estatinas no que tange a serem antioxidantes e antiinflamatórios e seus derivados retardam a neuro-inflamação, o estresse oxidativo e a morte de células apoptóticas. Além disso, inibem a formação de proteína $\beta$ amilóide. ${ }^{2}$

Outra possibilidade de ação dos AGP $\omega 3$ está na competição com o estoque de AA levando à inibição da produção de eicosanóides pró-inflamatórios. Podem também servir como substrato alternativo para a ciclo-oxigenase (COX e COX2) resultando na formação de produtos menos potentes que os pró-inflamatórios. ${ }^{27}$

O recrutamento dos Neutrófilos ocorre na fase inicial da inflamação e na sequiência ocorre um estímulo quimiotático que os direciona através das células do endotélio vascular. Este mecanismo é auxiliado pelo AA que é convertido em PTG-D2. Quando se disponibiliza um AGP $\omega 3$ para a célula, forma-se PTGD3 que inibe a migração dos Neutrófilos através da célula endotelial por antagonizar receptor de PTG-D2. Estas funções dos AGP $\omega 3$ sinalizam um novo caminho na pesquisa e produção de novas drogas antiinflamatórias.

\section{Recomendaçōes nutriclonais e efel- tos da suplementação com AGP}

A razão entre a ingestão diária de alimentos fontes de AGP $\omega 6$ e $\omega 3$ assume grande importância na nutrição humana, resultando em várias recomendações que têm sido estabelecidas por diferentes autores e órgãos de saúde em vários países. ${ }^{29}$ Suécia $^{30}$ e França ${ }^{31}$ têm estabelecido recomendações para uma ingestão por meio da dieta de $\omega 6$ e $\omega 3$, na razão de 5:1, enquanto o Japão ${ }^{32}$ estabelece uma relação de 2:1. A Organização Mundial da saúde (WHO) / Food and Agriculture Organization (FAO) estabelece uma ingestão de $\omega 6$ e $\omega 3$, na razão de 5:1 a 10:1.33

Com a industrialização e as mudanças no padrão alimentar da população ocorreu um aumento progressivo dessa razão, resultando em dietas com quantidades inadequadas de $\omega 3$. Nas últimas décadas temse determinado que a ingestão média de AGP resulta em relações de 6: 3 que atingem 20:1, ocorrendo registros de até 50:1. . $^{34,35}$

A Dietary Reference Intakes (DRI), ao invés da razão $\omega 6: \omega 3$, estabelece nível de ingestão para os ácidos graxos individualmente. A ingestão diária recomendada para os ácidos linoléico e linolênico varia conforme idade, gênero e estados fisiológicos, como gestação e lactação. Para homens de 19 a 50 anos a ingestão de $\omega 6$ deve ser de $17 \mathrm{~g} / \mathrm{dia}$ enquanto que, para mulheres da mesma idade, a ingestão deve corresponder a $12 \mathrm{~g} / \mathrm{dia}$. Quanto à ingestão de $\omega 3$ são recomendados $1,6 \mathrm{~g} /$ dia para homens e $1,1 \mathrm{~g} /$ dia para mulheres. ${ }^{36}$

Estudos mostram que a composição lipídica das células imunes de roedores possui aproximadamente 15 a $20 \%$ de AG como o AA e pequenas concentrações de AGP $\omega 3$. Quando a dieta destes animais é modificada em sua concentração de AG, também ocorre modificação na composição destes ácidos na membrana das células imunes. O que se observa é aumento na concentração do ácido que foi usado para enriquecer a dieta. Seres humanos que consomem uma dieta tida como normal possuem células imunes (monócitos, linfócitos e neutrófilos) com $20 \%$ de AA, $1 \%$ EPA e $2,5 \%$ de DHA. A suplementação da dieta com um determinado $\mathrm{AG}$ aumenta sua proporção na célula imune. $^{21}$

A incorporação de EPA e DHA na dieta pode influenciar a estrutura lipídica das membranas celulares e as respostas fisiológicas que dependem destas membranas como é o caso dos mecanismos de sinalização celular. Os AGP $\omega 3$ obtidos da dieta podem colaborar com a diminuição dos processos inflamatórios e diminuir a incidência de doenças relacionadas à inflamação. A conversão de ácido linoléico a EPA ocorre nos vegetais, mas sua conversão em DHA parece ser muito pequena em seres humanos. Os efeitos benéficos do ácido linoléico nos humanos parecem dever-se à sua conversão a EPA, mas como isto é limitado, o seu consumo não teria grandes repercussões na saúde do indivíduo. O ideal seria o consumo de uma associação de EPA e DHA., $22,23,37-39$

A suplementação destes $A G$ pode ser feita através do consumo de óleo de peixe ou uso de emulsões constituídas por óleo de coco, soja, oliva ou peixe (a recomendação para o consumo de peixe, segundo a American Dietetic Association/ Dietitians of Canada $^{40}$ é de $500 \mathrm{mg}$, duas vezes por semana). Estas emulsões foram utilizadas por vários autores em nutrição parenteral em pacientes hospitalizados e verificaram efeitos benéficos relacionados, possivelmente, à normalização das funções imunes das células e modulação das respostas inflamatórias. Isto mostra que os efeitos dos AG podem ser obtidos em pacientes que não conseguem se alimentar normalmente e precisam do suporte da alimentação parenteral. ${ }^{22,41-43}$ 


\section{Resolvinas e protectinas}

As resolvinas (termo relacionado aos produtos formados na fase de resolução) foram primeiramente descritas para indicar a formação de moléculas mediadoras com capacidade antiinflamatória e com propriedades imunomodulatórias, incluindo a redução de migração dos neutrófilos e citocinas pró-inflamatórias quanto diminuindo a resposta inflamatória in vivo. A designação de protectina ou neuroprotectina (quando formada no tecido nervoso) foi dada inicialmente em relação ao efeito anti-inflamatório dos mediadores resultantes de DHA nos sistemas neuronais, na doença de Alzheimer e no infarto. ${ }^{44,45}$

Fatores de crescimento e citocinas associamse aos seus receptores celulares específicos e podem desencadear a ativação de uma série de fosfolipases que agirão sobre os fosfolipídeos de membrana e resultarão em AA. A partir deste ácido serão formados vários precursores lipídicos com função de mediadores químicos por ação da Citocromo P450 monooxigenase em duas vias principais. A primeira é a via mediada pela COX e a segunda pela LOX. Os produtos resultantes destas vias enzimáticas vão depender de quais são os tipos celulares que estão funcionando como alvos. ${ }^{17,46}$

Os AGP $\omega 3$ são precursores de mediadores que incluem as resolvinas da série E (E1 ou RvE1: ácido 5S, 12R,18R-trihidroxi-6Z, 8E, 10E, 14Z, 16E eicosapentanóico / E2 ou RvE2: ácido 5S, 18 diidroxieiconadóide) que derivam dos EPA. Também são precursores das resolvinas da série $\mathrm{D}$ (D1 ou RvD1: ácido 7S, 8R, 17S-trihidroxi-4Z, 9E, 11E, 13Z, 15E, $19 \mathrm{Z}$ docosapentanóico / D2 ou RvD2: ácido 7S, 16R, 17Strihidroxi-docosa-4Z, 8E, 10Z, 12E, 14E, $19 \mathrm{Z}$ hexaenóico) e suas formas epiméricas desencadeadas por Aspirina ${ }^{\circledR}$ derivadas de DHA, e as protectinas $(\mathrm{PT}) /$ neuroprotectinas (NPD), também derivadas de DHA (Figura 2). A produção de RvE1 em homens saudáveis é aumentada após o uso de Aspirina ${ }^{\circledR}$, entretanto quantidades significantes de RvE1 em concentrações que promovem efeito biológico são encontradas no plasma mesmo sem a ingestão deste medicamento. . $^{74,47}$

Uma nova classe de mediadores lipídicos do tipo pró-resolvinas formadas a partir de DHA por macrófagos foi muito recentemente identificada. A descoberta desta substância foi feita a partir de estudos que utilizaram exsudatos colhidos de ratos sofrendo de peritonite. Neste material havia acúmulo de ácido $17-$ hidroxidocosanóico (da série de resolvinas D e de protectinas) e ácido 14S-hydroxi-docosa-4Z,7Z,10Z, 12E,16Z,19Z-hexaenóico que é derivado de DHA. A adição destas duas últimas substâncias a macrófagos originou produtos com forte atividade antiinflamatória e com função de pró-resolvina. A atividade destas substâncias, nominadas de maresinas, têm potencial próximo ao das resolvinas da série E1 e protectinas. A biossíntese das maresinas envolve uma via diferente de ação das lipoxigenases que geram produtos bioativos do tipo ácido 7,14-dihidroxidocosa-4Z,8,10,12, 16Z,19Z-hexaenóico. 27,46,48

O início e o fim do processo inflamatório são de extrema importância para o organismo. O início é imprescindível para que seja feita a defesa ao trauma ou ao microorganismo invasor e o término é necessário para que não sejam maximizadas as reações inflama-
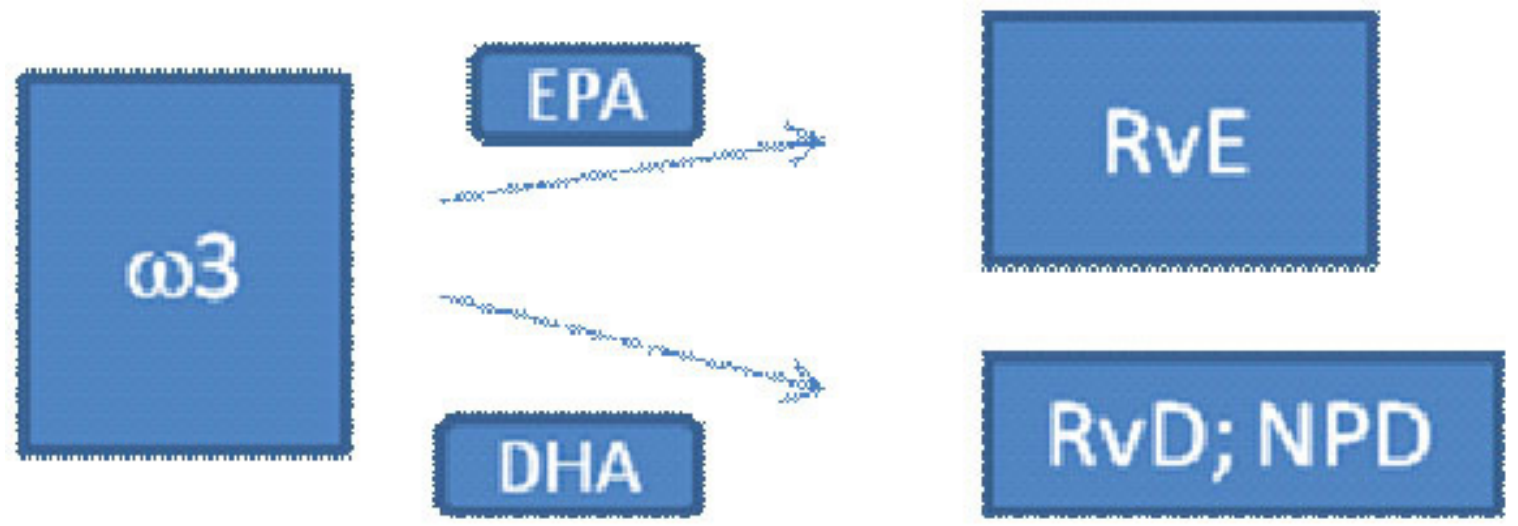

Figura 2. Formação de resolvinas $D$ e $E$ (RvD e RvE) e neuroprotectinas (NPD) a partir de EPA (resolvina E) e DHA (resolvina D). Adaptado de SERHAN, CHIANG, 2008. 
tórias e outros danos tissulares sejam desencadeados, o que culminaria em uma doença. Neste dueto, início/ fim estão envolvidas as mesmas substâncias lipídicas por levar à terminação por estimular a síntese de resolvinas. Sendo assim, o uso de AGP 13 (EPA e DHA) pode ser promissor na minimização ou prevenção de doenças de cunho e implicações inflamatórias como a obesidade, diabetes, asma, doenças inflamatórias intestinais e os sintomas de dor por ação central e periférica. ${ }^{28,43,49,50}$

\section{Locais de ação das resolvinas}

As resolvinas regulam a migração de neutrófilos os quais constituem a primeira linha de defesa do sistema imune. As RvE1 reduzem dramaticamente a infiltração destas células em áreas inflamadas o que é importante para a resolução da inflamação assim como na invasão destas áreas por patógenos. Se não há este tipo de resposta, pode ocorrer uma reação inflamatória excessiva, o que causa danos substanciais ao tecido. Também inibe a geração de superóxido e bloqueia a quimiotaxia via IL-8.2,50,51

Em Macrófagos as resolvinas promovem fagocitose de Neutrófilos (clearence de neutrófilos apoptóticos) e promove a migração de Macrófagos contendo zimozan de locais inflamados para os órgãos linfáticos. ${ }^{52}$ Em células dendríticas inibem a indução de TNF- $\alpha$ e NFK $\beta$ e a liberação de IL-6 e IL-2. ${ }^{2,51,53}$

As plaquetas são de extrema importância na coagulação sanguínea, na cura de ferimentos e nos processos inflamatórios. As RvE1 bloqueiam seletivamente o receptor de tromboxano e a agregação plaquetária excessiva mas não bloqueiam a agregação estimulada por colágeno. Portanto, as RvE1 têm efei- tos anti-plaquetários seletivos, o que leva a crer que o uso de EPA pode ser benéfico em seres humanos. ${ }^{54}$

As resolvinas também regulam as moléculas de adesão da superfície celular que são essenciais para a saída de leucócitos. RvE1 diminui a expressão de CD18 em Neutrófilos e Monócitos e modifica a ação da Lselectina (que está relacionada aos eventos iniciais do recrutamento de Leucócitos), resultando na redução da migração de Neutrófilos para o local da inflamação. $2,54-57$

As resolvinas são produzidas em resposta a um estímulo, têm ação local e têm metabolização por vias enzimáticas que levam à sua inativação. As RvE1 podem ser convertidas a diferentes metabólitos (20hidroxi-RvE1, 20-carboxi-RvE1, 19-hidroxi-ReV1, 18oxo-RvE1 e 10, 11-diidro-RvE1) em Neutrófilos humanos que são biologicamente inativos se comparados às RvE1. 2,48,55-57

\section{Conclusões}

As doenças inflamatórias têm papel importante na saúde pública já que estão relacionadas com a redução da qualidade de vida e a altos custos para os sistemas de saúde. Os medicamentos utilizados têm alto custo para os pacientes e muitas vezes estão relacionadas a múltiplos efeitos adversos. As resolvinas, protectinas e maresinas, recentemente descritas, são derivadas de AGP $\omega 3$ e têm mostrado efeitos importantes na minimização dos processos inflamatórios. Sendo assim, pode-se dizer que o estudo destas substâncias e o delineamento de seus efeitos podem trazer novos horizontes no tratamento das doenças inflamatórias, com redução de custos e minimização de efeitos colaterais.

\begin{abstract}
Inflammation is a defensive response to the trauma, infections or injury to the organism. When inflammation is ending occurs a program for active regulation called the resolution. This is accompanied by a class of lipid mediators where there is alternation of pro-inflammatory prostaglandins and leukotrienes to proresolution mediators, produced from 3 polyunsaturated fatty acids (eicosapentaenoic and docosapentanoic acid). The diet interferes the fatty acid composition of cell membranes that will be metabolized to active compounds called resolvins, protectins and maresins. The inflammatory diseases have an important role in public health, so the study of these substances and delineation of their effects can bring new horizons in the treatment of inflammatory diseases, reducing costs and minimizing side effects.
\end{abstract}

Key Words: Inflammation. Fatty Acids, Omega-3. Resolvins. Protectins. 


\section{Referências Bibliográficas}

1. Calder PC. N-3 polyunsaturated fatty acids, inflammation and inflamatory diseases. Am J Clin Nutr 2006 (83): 1505S-19S.

2. Seki H, Tani Y, Arita M. Omega3 PUFA derived anti-inflammatory lipid mediator resolving E1. Prostaglandins other lipid mediat 2009; 89:126-30.

3. Voltarelli JC. Febre e inflamação. Medicina (Ribeirão Preto) 1994; 27: 7-48.

4. Vannuchi $\mathrm{H}$, Moreira EAM, Cunha DF, Junqueira-Franco MVM, Bernardes MM; Jordão-Jr AA. Papel dos nutrientes na peroxidação lipídica e no sistema de defesa antioxidante. Medicina Ribeirão Preto 1998; 31: 31-44.

5. Cardoso, LM, Colombari DAS, Menani JV, De Paula PM, Chianca DA, Colombari E. Espécies reativas de oxigênio no controle neurovegetativo da pressão arterial. Medicina (Ribeirão Preto) 2006 ; 39: 77-88.

6. Flower RJ. Prostaglandins, bioassay and inflammation. $\mathrm{Br} \mathrm{J}$ Pharmacol 2006; 147 (Suppl 1): 182-92.

7. Serhan CN, Chiang N. Endogenous pro-resolving and antiinflammatory lipid mediators: a new pharmacologic genus. Br J Pharmacol 2008;153 (Suppl 1): 200-15.

8. Dominguez-Rodriguez A, Abreu-Gonzalez P, Kaski JC. Inflammatory systemic biomarkers in setting acute coronary syndromes--effects of the diurnal variation. Curr Drug Targets 2009; 10:1001-8.

9. Beavers KM, Brinkley TE, Nicklas BJ. Effect of exercise training on chronic inflammation. Clin Chim Acta 2010; 411:78593.

10. Spite M, Norling LV, Summers L, Yang R, Cooper D, Petasis NA, Flower RJ, Perretti M, Serhan CN. Resolvin D2 is a potent regulator of leukocytes and controls microbial sepsis. Nature 2009; 461:1287-91.

11. Chan MM, Moore AR. Resolution of inflammation in murine autoimmune arthritis is disrupted by cyclooxygenase-2 inhibition and restored by Prostaglandin E(2)-mediated lipoxin A(4) production. J Immunol 2010; 184:6418-26.

12. Persson CG, Uller L. Resolution of cell-mediated airways diseases. Respir Res 2010; 11:75.

13. Levy BD. Resolvins and protectins: natural pharmacophores for resolution biology. Prostaglandins Leukot Essent Fatty Acids 2010; 82: 327-32.

14. Devchand PR, Schmidt BA, Primo VC, Zhang QY, Arnaout MA, Serhan CN, Nikolic B. A synthetic eicosanoid LX-mimetic unravels host-donor interactions in allogeneic BMT-induced GvHD to reveal an early protective role for host neutrophils. Faseb J 2005; 19:203-10

15. Arita M, Bianchini F, Aliberti J, Sher A, Chiang N, Hong S, Yang R, Petasis NA, Serhan CN. Stereochemical assignment, antiinflammatory properties, and receptor for the omega-3 lipid mediator resolvin E1. J Exp Med 2005; 201:713-22.

16. Bueno-Jr CRB. Relevância e tratamento das lesões características da insuficiência cardíaca na musculatura esquelética. Medicina Ribeirão Preto 2009; 42: 437-50.

17. Hersberger M. Potential role of the lipoxygenase derived lipid mediators in atherosclerosis: leukotrienes, lipoxins and resolvins. Clin Chem Lab Med 2010; 48: 1063-73.

18. Gilroy DW, Lawrence T, Perrettti M, Rossi AG. Inflammation, resolution: new opportunities for drug discovery. Nat Rev Drug Discov 2004; 3: 401-16.
19. Tull SP, Yates CM, Maskrey BH, O'Donnell VB, Madden J, Grimble RF, Calder PC, Nash GB, Rainger GE. Omega-3 Fatty acids and inflammation: novel interactions reveal a new step in neutrophil recruitment. Plos Biol 2009; 7: e1000177.

20. Hunter JE, Zhang J, Kris-Etherton PM. Cardiovascular disease risk of dietary stearic acid compared with trans, other saturated, and unsaturated fatty acids: a systematic review. Am J Clin Nutr 2010; 91: 46-63.

21. Calder, PC. Immunomodulation by omega 3 fatty acids. Prost Leukotrienes and essential fatty acids 2007; 77: 327-35.

22. Calder PC. Polyunsaturated fatty acids and inflammatory processes: New twists in an old tale. Biochimie 2009; 91:791-5.

23. Calder PC, Yaqoob P. Understanding omega-3 polyunsaturated fatty acids. Postgrad Med 2009; 121:148-57.

24. Calder PC, Yaqoob P.Omega-3 polyunsaturated fatty acids and human health outcomes. Biofactors 2009; 35:266-72.

25. Lund T, Mangsbo SM, Scholz H, Gjorstrup P, Tötterman TH, Korsgren O, Foss $A$. Resolvin E1 reduces proinflammatory markers in human pancreatic islets in vitro. Exp Clin Endocrinol Diabetes 2010; 118: 237-44.

26. He K, Song Y, Daviglus ML, Liu K, Van Horn L. Accumulated evidence of fish consumption and coronary heart disease mortality: a meta-analysis of cohort studies. Circulation 2004; 109: 2705-11.

27. Serhan CN, Yang R, Martinod K, Kasuga K, Pillai PS, Porter TF, Oh SF, Spite M. Maresins: novel macrophage mediators with potent antiinflammatory and proresolving actions. J Exp Med 2009; 206:15-23.

28. Seki H, Sasaki T, Ueda T, Arita M. Resolvins as regulators of the immune system. Scientific World Journal 2010; 10:81831.

29. Martin CA, Almeida VV, Ruiz MR, Visentainer JEL, Matshushita M, Souza NE, Visentainer JV. Ácidos graxos poliinsaturados ômega-3 e ômega-6: importância e ocorrência em alimentos. Rev. Nutr 2006; 19:761-70.

30. Nordic Council of Ministers. Nordic nutrition recommendations. Scand J Nutr 1996; 40:161-5.

31. Chardigny JM, Bretillon L, Sébédio JL. New insights in health effects of trans alpha-linolenic acid isomers in humans. Eur J Lipid Sci Technol 2001; 103:478-82.

32. Kris-Etherton PM, Taylor DS, Yu-Poth S, Huth P, Moriarty K, Fishell V, et al. Polynsaturated fatty acids in the food chain in the United States. Am J Clin Nutr 2000; 71 (Suppl 1):179-88.

33. World Health Organization. Joint Consultation: fats and oils in human nutrition. Nutr Rev 1995; 53:202-5.

34. Simopoulos AP. Omega-6/Omega-3 essential fatty acid ratio and chronic diseases. Food Rev Inter 2004; 20:77-90.

35. Simopoulos AP. Omega-3 fatty acids in wild plants, nuts and seeds. Ásia Pacific J Clin Nutr 2002; 11:163-73.

36. Institute of Medicine. National Research Council. Dietary Intakes for energy, carbohydrate, fiber, fat, fatty acids, cholesterol, protein, and amino acids (macrontrients). Washington (DC): National Academy Press; 2002.

37. Poppitt SD, Howe CA, Lithander FE, Silvers KM, Lin RB, Croft J, Ratnasabapathy Y, Gibson RA, Anderson CS. Effects of moderate-dose omega-3 fish oil on cardiovascular risk factors and mood after ischemic stroke: a randomized, controlled trial. Stroke 2009; 40:3485-92.

38. Rupp H. Omacor (prescription omega-3-acid ethyl esters 90): From severe rhythm disorders to hypertriglyceridemia. Adv Ther 2009; 26:675-90. 
39. Bouwens M, Van De Rest O, Dellschaft N, Bromhaar MG, De Groot LC, Geleijnse JM, Müller M, Afman LA. Fish-oil supplementation induces antiinflammatory gene expression profiles in human blood mononuclear cells. Am J Clin Nutr 2009; 90:415-24.

40. Kris-Etherton PM, Innis S, Ammerican Dietetic Assocition, Dietitians of Canada. Position of the American Dietetic Association and Dietitians of Canada: dietary fatty acids. J Am Diet Assoc 2007; 107:1599-611.

41. Wanten GJ, Calder PC. Immune modulation by parenteral lipid emulsions. Am J Clin Nutr 2007; 85:1171-84.

42. Jiang ZM, Wilmore DW, Wang XR, Wei JM, ZhanG ZT, Gu ZY, Wang S, Han SM, Jiang H, Yu K. Randomized clinical trial of intravenous soybean oil alone versus soybean oil plus fish oil emulsion after gastrointestinal cancer surgery. $\mathrm{Br} \mathrm{J}$ Surg 2010; 97:804-9.

43. Levy BD, Lukacs NW, Berlin AA, Schimidt B, Guilford WJ, Serhan CN. Lipotoxin A4 anlogs reduce allergic airway responses via mechanisms distinct from CysLT1 receptor antagonism. Faseb J 2007; 21: 3877-84.

44. Marcheselli VL, Hong S, Lukiw WJ, Hua Tian X, Gronert K. Novel docosanoids inhibit brain ischemia-reperfusion-mediated leukocyte infiltration and pro-inflammatory gene expression. J Biol Chem 2003; 278: 43807-17.

45. Lukiw WJ, Cui JG, Marcheselli VL, Bodker M, Botkjaer A. A role for docosahexaenoic acid-derived neuriprotectin D1 in neural cell survival and Alzheimer disease. J Clin Invest 2005; 115: 2774-83.

46. Norling LV, Serhan CN. Profiling in resolving inflammatory exudates identifies novel anti-inflammatory and pro-resolving mediators and signals for termination. J Intern Med 2010; 268:15-24.

47. Chiang N, Serhan CN, Dahlén SE, Drazen JM, Hay DW, Rovati GE, Shimizu T, Yokomizo T, Brink C. The lipoxin receptor ALX: potent ligand-specific and stereoselective actions in vivo. Pharmacol Rev 2006; 58:463-87.
48. Hong S, Porter TF, Lu Y, Oh SF, Pillai PS, Serhan CN. Resolvin E1 metabolome in local inactivation during inflammation resoIution. J Immunol 2008; 180: 3512-9.

49. González-Périz A, Clària J. Resolution of adipose tissue inflammation. Scientific World Journal 2010; 10:832-56.

50. Xu ZZ, Zhang L, Liu T, Park JY, Berta T, Yang R, Serhan CN, Ji RR. Resolvins RvE1 and RvD1 attenuate inflammatory pain via central and peripheral actions. Nat Med 2010; 16:592-7.

51. Farooqui AA, Ong WY, Horrocks LA, Chen P, Farooqui T. Comparison of biochemical effects of statins and fish oil in brain: the battle of the titans. Brain Res Rev 2007; 56: 44371.

52. Shwab JM,Chiang N, Arita M, Serhan CN. Resolvin E1 and protectin D1 activate inflammation-resolution programmes. Nature 2007; 447: 869-74

53. Coelho-Castelo AAM, Trombone APP, Rocha CD, Lorenzo JCC. Resposta imune a doenças infecciosas. Medicina (Ribeirão Preto) 2009; 42:127-142.

54. Dona M, Fredman G, Schwab JM, Chiang N, Arita M, Goodarzi A, Cheng G, VON Andrian UH, Serhan CN.Resolvin E1, an EPA-derived mediator in whole blood, selectively counterregulates leukocytes and platelets. Blood 2008; 112:848-55.

55. Maderna P, Godson C. Lipoxins: resolutionary road. $\mathrm{Br} \mathrm{J}$ Pharmacol 2009; 158:947-59.

56. Morris T, Stables M, Colville-Nash P, Newson J, Bellingan G, De Souza PM, Gilroy DW. Dichotomy in duration and severity of acute inflammatory responses in humans arising from differentially expressed proresolution pathways. Proc Natl Acad Sci U S A 2010; 107:8842-7.

57. Seki H, Fukunaga K, Arita M, Arai H, Nakanishi H, Taguchi R, Miyasho T, Takamiya R, Asano K, Ishizaka A, Takeda J, Levy $B D$. The anti-inflammatory and proresolving mediator resolvin E1 protects mice from bacterial pneumonia and acute lung injury. J Immunol 2010; 184:836-43. 